Supporting Information

\title{
Detection and Separation of Single-stranded DNA \\ Fragments using Solid-state Nanopores
}

Lijian Zhan ${ }^{\dagger}$, Yin Zhang ${ }^{\dagger, *}$, Wei Si, Jingjie Sha and Yunfei Chen*

Jiangsu Key Laboratory for Design and Manufacture of Micro-Nano Biomedical Instruments, School of Mechanical Engineering, Southeast University, Nanjing

211189, China

\section{S1. Nanopore fabrication.}

First of all, the $1 \mu \mathrm{m}$ thick $\mathrm{SiO}_{2}$ layers were grown on both sides of a $200 \mu \mathrm{m}$ thick $\mathrm{Si}$ substrate (RDMICRO Inc.) by wet oxidation. The $60 \mathrm{~nm}$ thickness low stress $\mathrm{Si}_{3} \mathrm{~N}_{4}$ membranes were deposited on the $\mathrm{SiO}_{2}$ dielectric layers by low pressure chemical vapor deposition (LPCVD). Then a $2 \mu$ m diameter circular region was etched on the top $\mathrm{Si}_{3} \mathrm{~N}_{4}$ membrane with a depth of $30 \mathrm{~nm}$ by reactive ion etching (RIE). After that, optical lithography was used to pattern a square etch window at the center of the other side of the silicon wafer. Finally, the wafer went through a potassium hydroxide $(\mathrm{KOH})$ wet etching process to remove Si substrate and expose a free-standing $\mathrm{Si}_{3} \mathrm{~N}_{4}$ membrane. The etched region on the free-standing membrane was characterized by atomic force microscopy (AFM) and shown in Figure S1a. Figure S1b shows the depth of etched region, $\Delta h$, measured by AFM in contact model. So we could easily calculate the thickness of $\mathrm{Si}_{3} \mathrm{~N}_{4}$ membrane

$$
h_{m}=60 \mathrm{~nm}-\Delta h=30 \mathrm{~nm}
$$

Then, the dielectric controlled breakdown (CBD) method ${ }^{1-3}$ was used to fabricate a nanopore on the free-standing membrane as illustrated in Figure S1c. The voltage 
pulse with $25 \mathrm{~Hz}$ was applied by a computer-controlled source meter (2612A, Keithley). And the ionic current across the membrane was detected by the source meter to verify whether a nanopore was generated. Figure S1d shows the measured I-V curves in the condition of $1 \mathrm{M} \mathrm{KCl}$. The value of nanopore diameter can be calculated from the measured pore conductance, $G$, according to Equation 2.4-5

$$
G=\kappa\left(\frac{4 L}{\pi d^{2}}+\frac{1}{d}\right)^{-1}
$$

Where $\kappa$ is the bulk ion conductivity of the solution (for $1 \mathrm{M} \mathrm{KCl,} \kappa=11.18 \mathrm{~S} / \mathrm{m}$ at $25^{\circ} \mathrm{C}$ and $\mathrm{pH} 7^{6}$ ), $L$ is the nanopore length, and $d$ is the effective nanopore diameter. The calculated nanopore diameters by Equation 2 are $d=5.4 \mathrm{~nm}, \mathrm{~d}=8.2 \mathrm{~nm}$, and $\mathrm{d}=11.2 \mathrm{~nm}$, respectively.

(a)

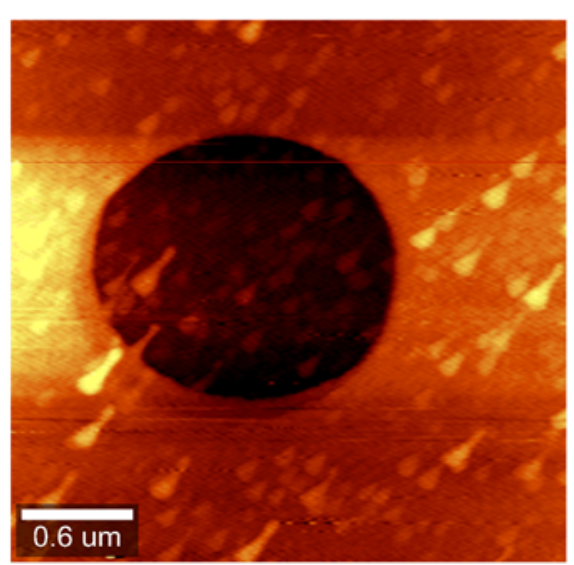

(c)

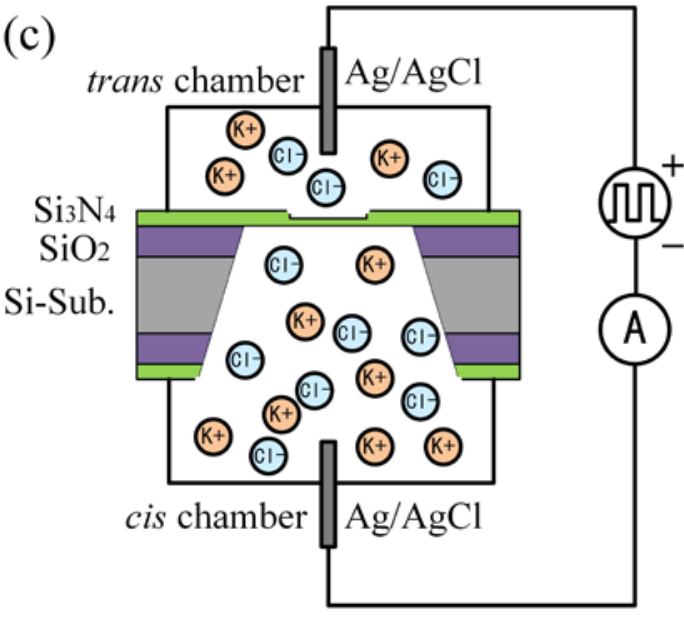

(b)

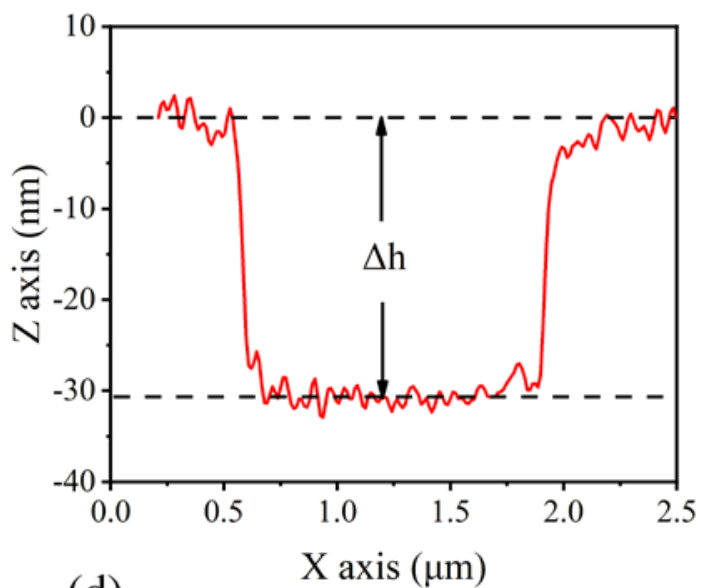

(d)

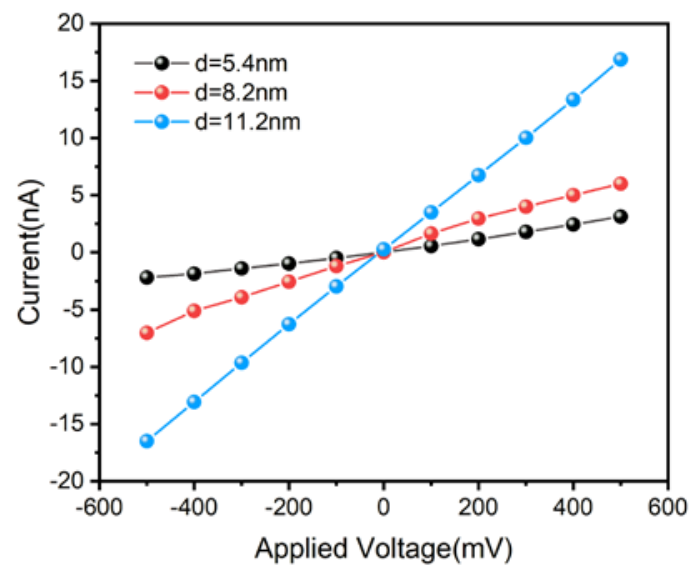


Figure S1. Nanopore fabrication (a) A circular etched region was characterized by AFM. (b) Scanning height profile of the etched region. (c) Schematic of the CBD fabrication setup. Two Ag/AgCl electrodes were immersed in Cis and trans chambers and connected to a source meter. (d) The I-V curves measured by Axon MultiClamp 700B through sweeping the applied bias voltage from $-500 \mathrm{mV}$ to $+500 \mathrm{mV}$ with a step of $100 \mathrm{mV}$. And the diameters of nanopores calculated by Equation 2 are respectively $5.4 \mathrm{~nm}$ (black point), $8.2 \mathrm{~nm}$ (red point), and $\mathbf{1 1 . 2}$ nm (blue point). 


\section{S2. Experimental procedure.}

In our experiments, the chips with nanopore were processed by standard cleaning procedures. Firstly, the nanopore chip was cleaned with piranha solution (3:1 $\mathrm{H}_{2} \mathrm{SO}_{4} / \mathrm{H}_{2} \mathrm{O}_{2}$ ) at $120{ }^{\circ} \mathrm{C}$ for 40 min to remove organic contamination and followed by deionized repeated water rinsing. Then, the chip was clamped between two silicone elastomer gaskets conglutinated at the mouth of a polymethylmethacrylate flow-cell, which filled with $1 \mathrm{M} \mathrm{KCl}$ electrolyte solution. In addition, the $\mathrm{pH}$ of $\mathrm{KCl}$ electrolyte solution was adjust to $\sim 10$ by $\mathrm{KOH}$ titration at ambient temperature condition to enhance the surface charge density. Two Ag/AgCl electrodes were immersed into two respective electrolyte compartments for setting up a transmembrane potential and detecting the ionic signals. Current traces were measured by a resistive feedback amplifier (Axon MultiClamp 700B) at a sampling rate of $250 \mathrm{kHz}$ and filtered using a $10 \mathrm{kHz}$ low-pass filter, inside a dark Faraday cage.

All types of ssDNA fragments used in experiments were purchased from Sangon Biotech Co., Ltd. The sequences (5' to 3') of 20nt, 50nt and 90nt ssDNA are respectively presented in below

20nt: ATC GAC CGT AAT CGA CCG TA

50nt: ATC GAC CGT AAT CGA CCG TAA TCG ACC GTA ATC GAC CGT AAT CGA CCG TA

90nt: ATC GAC CGT AAT CGA CCG TAA TCG ACC GTA ATC GAC CGT AAT CGA CCG TAA TCG ACC GTA ATC GAC CGT AAT CGA CCG TAA TCG ACC GTA.

The 50nt and 90nt ssDNAs were purified by high-performance liquid chromatography 
(HPLC) and 20nt ssDNAs were purified by polyacrylamide gel electrophoresis (PAGE). The results of mass spectrum identification of ssDNA samples are shown in Figure S2 a-c.

Before use, the ssDNA samples were steadily heated up to $60{ }^{\circ} \mathrm{C}$ in water bath and dramatically cooled to freezing point in ice bath. It could efficiently disperse ssDNA uniformly in electrolyte solution and enhance discreteness of current blockades. The current blockades corresponding to ssDNA translocation events were detected by a resistive feedback amplifier (Axon MultiClamp 700B). In our experiments, the same pore was used to measure three different lengths of ssDNAs. After the detection of one kind of ssDNAs, the chambers were washed by deionized water three times and monitored the ionic current without any pulse signals for about 10 min to make sure no ssDNA remained in the chamber. Then, the next ssDNA sample was added into the cis chamber and repeated the previous experimental processes.

(a)

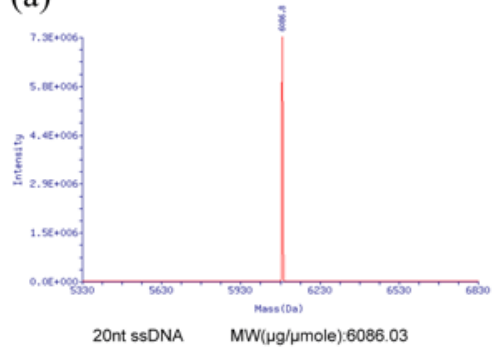

(b)

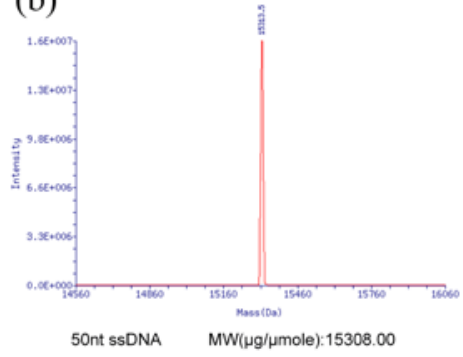

(c)

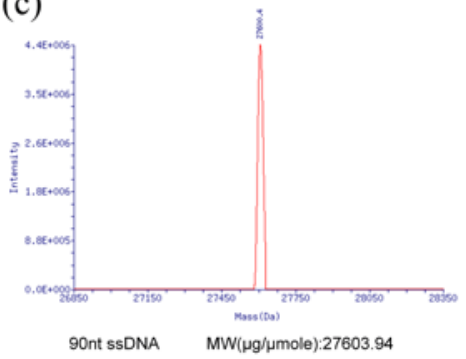

Figure S2. Results of mass spectrum identification of 20nt (a), 50nt (b), and 90nt (c) ssDNA samples, respectively. 


\section{S3. Length-dependent translocation of ssDNA under $\pm 400 \mathrm{mV}$.}

We fabricated another three nanopores with $5.7 \mathrm{~nm}, 8.5 \mathrm{~nm}$, and $11.6 \mathrm{~nm}$ diameter using CBD method, respectively. Figure S3a-c show the current traces of 20nt, 50nt and 90nt ssDNA translocation through $5.7 \mathrm{~nm}, 8.5 \mathrm{~nm}$ and $11.6 \mathrm{~nm}$ diameter nanopores under trans-membrane voltages of $\pm 400 \mathrm{mV}$, respectively. The current traces also reveal the length-dependent translocation behaviors of ssDNA.
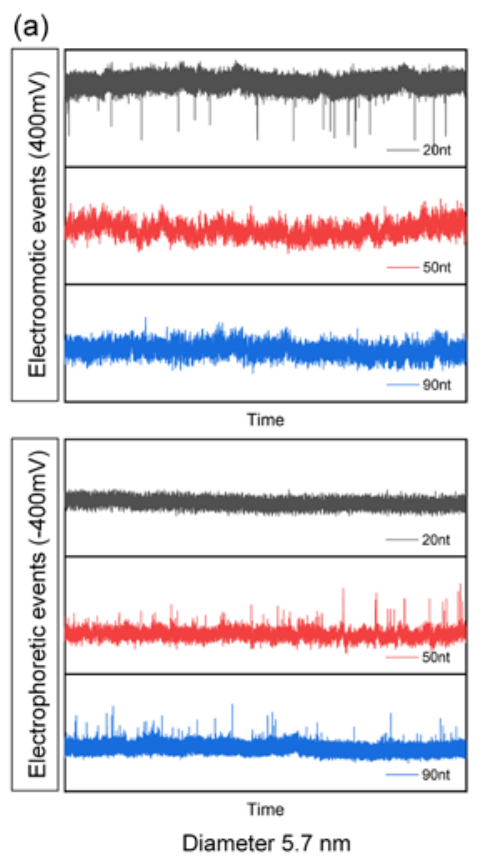
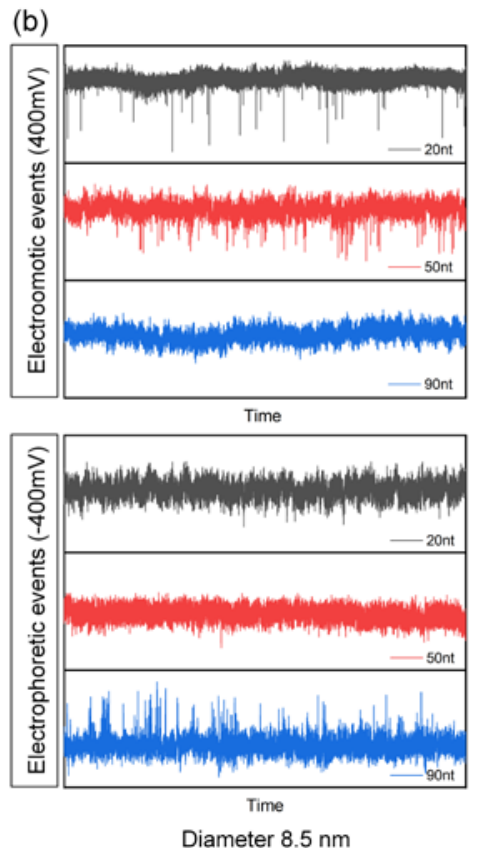

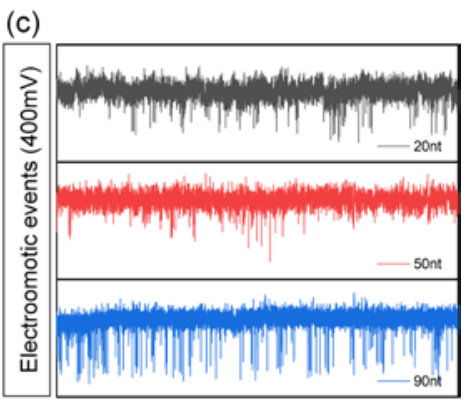

Time

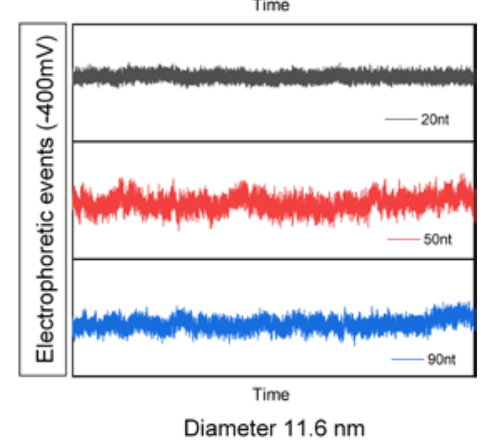

Figure S3. Ionic current traces with the translocation of 20nt (black), 50nt (red) and 90nt

(blue) ssDNA molecules through $5.7 \mathrm{~nm}$ (a), $8.5 \mathrm{~nm}$ (b) and $11.6 \mathrm{~nm}$ (c) diameter nanopores, respectively. The ssDNAs were added into cis chamber which filled with $1 \mathrm{M} \mathrm{KCl}$ solution at pH 10. The applied voltage on cis chamber was $+400 \mathrm{mV}$ or $-400 \mathrm{mV}$. 


\section{S4. Numerical simulation model.}

A series of equations were employed to describe the potential and ionic concentration distributions, fluid flow, ionic transport, and DNA translocation in the nanopore. ${ }^{7-8}$

The Poisson-Boltzmann equation for the ionic charge distribution in the liquid inside the nanopore

$$
\nabla^{2} \emptyset=-\frac{Z_{i} e \rho_{i}}{\epsilon \epsilon_{0}}=-\left(\frac{Z_{i} e \rho_{0}}{\epsilon \epsilon_{0}}\right) \exp \left(-Z_{i} e \emptyset / k T\right)
$$

$\emptyset$ is the electrical potential, $\rho_{i}$ is the number concentration of $i$ th ionic species, $\rho_{0}$ is the bulk number concentration of that species, and $Z_{i}$ is the ion valency.

The Navier-Stokes equation for the fluid motion

$$
\rho\left(\frac{\partial \vec{v}}{\partial t}+\vec{v} \cdot \nabla \vec{v}\right)=-\nabla p+\mu \nabla^{2} \vec{v}+\vec{F}
$$

$\vec{v}$ is the velocity of the liquid, $p$ is the hydrostatic pressure, $\mu$ is the fluid viscosity, and $\mathrm{F}$ is the density of external forces on liquid.

The Nernst-Plank equation for ion motion in the liquid

$$
\frac{\partial \rho_{i}}{\partial t}=-\nabla \vec{N}_{i}=-\left(n_{i} \vec{v}-D_{i} \nabla \rho_{i}-\mu_{i} \rho_{i} \nabla \emptyset\right)
$$

$\vec{N}_{i}$ is the ionic flux density of the ith ionic species, $D_{i}$ is the diffusivity, and $\mu_{i}$ is the mobility.

Figure S4 illustrates the numerical simulation model including ssDNA, nanopore wall, and chambers. Parameters used in the calculation are shown in Table S1. 


\section{Simulation model (2D rotational symmetric model)}

\section{Enlarge view}

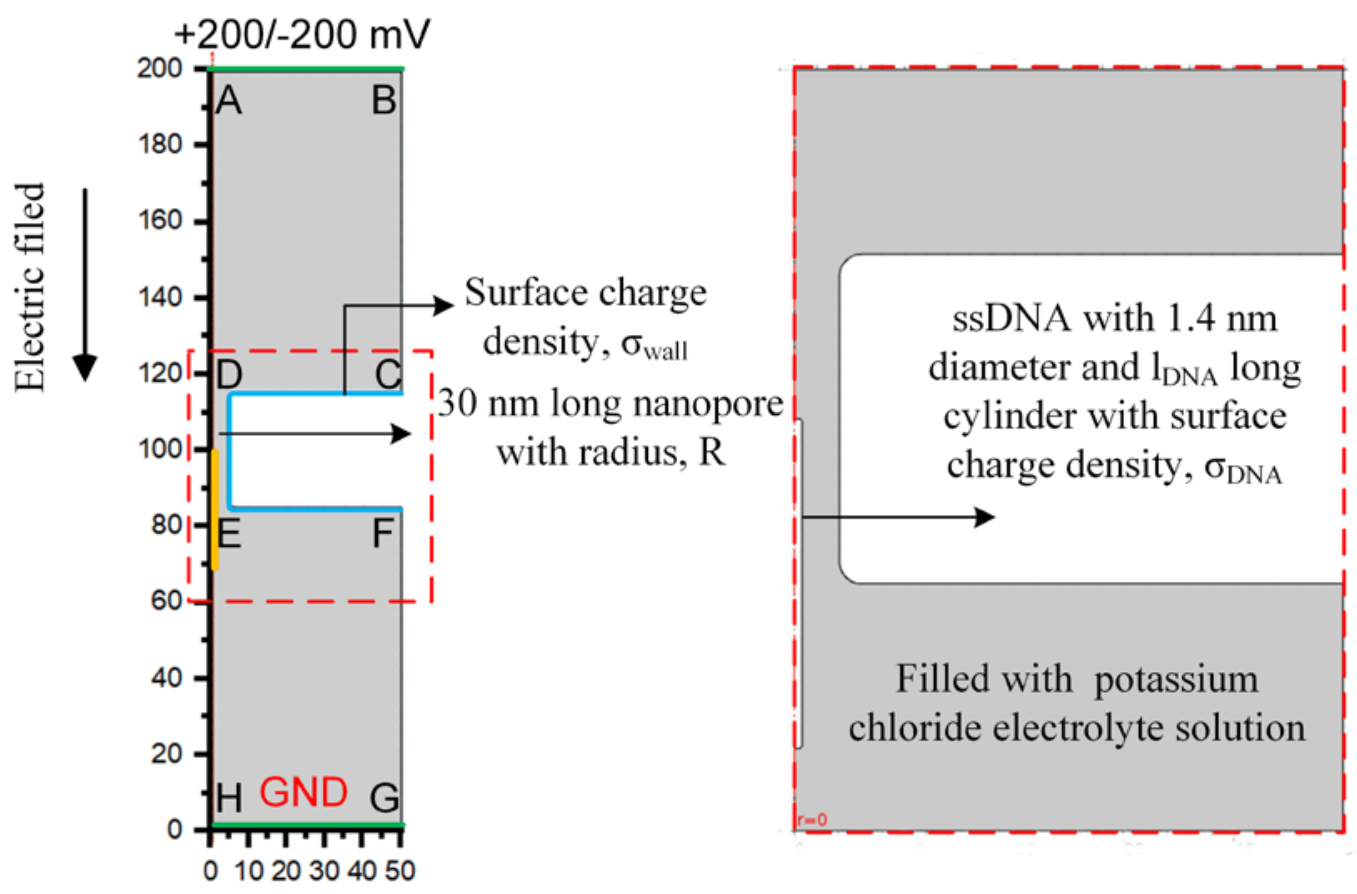

Figure S4. Numerical simulation model. The model was built in 2D rotational symmetric structure. The top green line (AB) represents the area where was applied bias voltage and the bottom one (GH) was ground; the electric potential drop was imposed between ( $\mathrm{AB}$ ) and (GH).

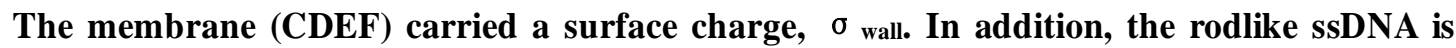
illustrated by yellow line and described at the enlarge view in detail.

Table S1. The fundamental parameters of simulation model.

\begin{tabular}{lll}
\hline Parameter & Value & Description \\
\hline r_DNA & $0.7 \mathrm{~nm}$ & Radius of ssDNA \\
\hline D1 & $1.957 \mathrm{e}-9\left[\mathrm{~m}^{2} / \mathrm{s}\right]$ & Diffusivity of $\mathrm{K}^{+}$ \\
\hline D2 & $2.03 \mathrm{e}-9[\mathrm{~m} 2 / \mathrm{s}]$ & Diffusivity of $\mathrm{Cl}^{-}$ \\
\hline C_bulk & $1000[\mathrm{mM}]$ & Bulk concentration \\
\hline V_pore & $200[\mathrm{mV}]$ & Applied bias voltage \\
\hline per_fluid & 80 & Relative permittivity of fluid \\
\hline per_vac & $0.85 \mathrm{e}-12[\mathrm{~F} / \mathrm{m}]$ & Permittivity of vacuum \\
\hline
\end{tabular}




\begin{tabular}{lll}
$T$ & $300[\mathrm{~K}]$ & Temperature \\
\hline eta & $1.0 \mathrm{e}-3[\mathrm{~Pa} \cdot \mathrm{s}]$ & Fluid viscosity \\
\hline Z_DNA & variate & Position of ssDNA \\
\hline
\end{tabular}




\section{S5. Surface charge estimation.}

In our experiments, the key parameters were the surface charge densities of ssDNA, $\sigma_{D N A}$ and nanopore inner face, $\sigma_{w a l l}$. Reporting in previous researches, the surface charge of $\mathrm{Si}_{3} \mathrm{~N}_{4}$ membrane in solution was dominated by the charged silanol ( $\left.\mathrm{SiO}^{-}\right)$ groups, which had chemical reaction with proton through following equilibrium. .-10 $^{9}$

$$
\mathrm{SiOH} \rightleftharpoons \mathrm{SiO}^{-}+\mathrm{H}^{+}
$$

Behrens and Grier ${ }^{11}$ developed a model for describing the diffuse layer potential, $\zeta$. Through considering the dissociation of $\mathrm{SiO}^{-}$groups of equilibrium constant, $p K$, and the Stern layer of capacitance, $C$, the $\zeta$ is yielded the following relation.

$$
\zeta\left(\sigma_{\text {wall }}\right)=\frac{\mathrm{kT}}{\mathrm{e}} \ln \left(\frac{-\sigma_{\text {wall }}}{\mathrm{e} \Gamma+\sigma_{\text {wall }}}\right)+\frac{\ln (10)}{\mathrm{e} / \mathrm{kT}}(\mathrm{pK}-\mathrm{pH})-\frac{\sigma_{\text {wall }}}{\mathrm{C}}
$$

where $k$ is Boltzmann constant, $T$ is the absolute temperature, $e$ is the fundamental electronic charge and $\Gamma$ is the surface density of chargeable sites. A second relation between $\zeta$ and $\sigma_{\text {wall }}$ derives from the charge and potential distribution in the diffuse layer. For the case where $d \gg \kappa^{-1}$ ( $\kappa^{-1}$ is the Debye screening length), no double layer overlap occurs, and the surface charge density is approximated using the Grahame equation.

$$
\sigma_{\text {wall }}(\zeta)=\frac{2 \epsilon \epsilon_{0} \mathrm{kT} \kappa}{\mathrm{e}} \sinh \left(\frac{\mathrm{e} \zeta}{2 \mathrm{kT}}\right)
$$

where $\epsilon$ is relative permittivity, $\epsilon_{0}$ is permittivity of vacuum. On basics of reported value for the parameters in chemical equilibrium model have ranges for $\Gamma=$ $5 \sim 8 \mathrm{~nm}^{-2}, p K=6 \sim 8.5$, and $C=0.2 \sim 2.9 \mathrm{~F} / \mathrm{m}^{2} .12-13$ Solving Equation 7 and Equation 8 yields a value for the surface charge $\sigma_{\text {wall }}=-29 \mathrm{mC} / \mathrm{m}^{2}$, at $\mathrm{pH}=10$.

It is well known that the surface charge of ssDNA comes from the phosphate group 
skeleton hydrolysis. And each phosphate group ideally dissociates a proton to appear a negative net charge. We assume that ssDNA bears uniform surface charge density and approximate ssDNA to a rodlike bar with $1.4 \mathrm{~nm}$ diameter. The distance between two adjacent bases in ssDNA expands to $\sim 0.6 \mathrm{~nm}^{14}$ because of unwinding and stretching. Therefore, surface charge density of ssDNA can be obtained by dividing the total net charge of ssDNA by the surface area. And the calculated surface charge density of ssDNA is $\sigma_{D N A}=-60 \mathrm{mC} / \mathrm{m}^{2}$. 


\section{S6. Electric field intensity and EOF velocity profiles inside nanopore.}

Figure S5a shows the electric field intensity profiles in different sized nanopore. With the pore size enlarging, the electric field intensity inside the nanopore obviously becomes smaller. Figure S5b shows the electric field intensity along the axis of different sized nanopores. It is well known the total resistance of nanopore in solution is equal to the sum of access resistances (inlet and outlet of nanopore) and resistance inside the pore. The proportion of access resistance in total resistance goes up with pore size. As a result, electric field intensity increases at the entrance but decreases inside the nanopore as pore size increases.

Figure S5c shows the EOF velocity profiles for the nanopores with radius of 1.5 $\mathrm{nm}, 5.0 \mathrm{~nm}$ and $7.5 \mathrm{~nm}$ when a ssDNA located in center of the pore. Interestingly, the EOF velocity inside the pore is not monotonically decreases with pore size enlarging. We also computed the EOF velocity profiles along nanopore radial direction for different nanopores. As shown in Figure S5d, the maximum of EOF velocity inside each sized nanopore presents a non-monotonic trend. We think it can be attributed to the concentration of counterions between nanopore inner face and ssDNA surface does not decrease to bulk concentration in the extremely small sized nanopore. So the EOF velocity in the extremely small sized nanopore cannot be fully-developed and smaller than velocity of EOF in relatively large sized pore. 
(a)

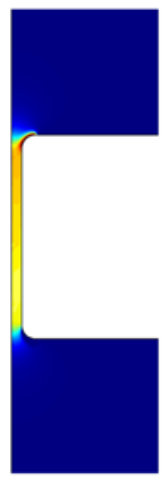

$\mathrm{r}=1.5 \mathrm{~nm}$

(c)

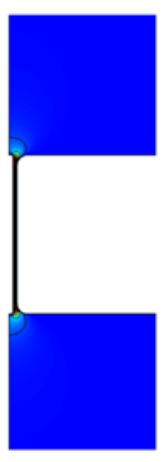

$\mathrm{r}=1.5 \mathrm{~nm}$

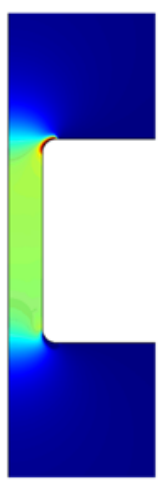

$\mathrm{r}=5.0 \mathrm{~nm}$

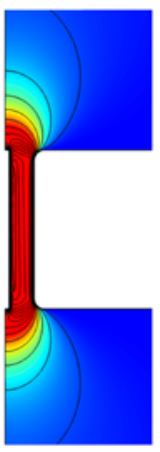

$\mathrm{r}=5.0 \mathrm{~nm}$

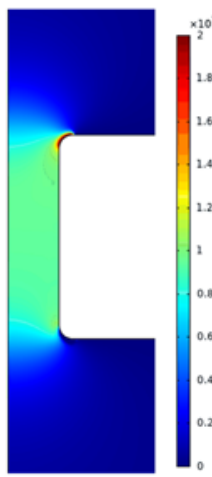

$\mathrm{r}=7.5 \mathrm{~nm}$

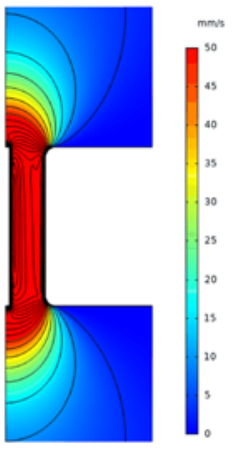

$\mathrm{r}=7.5 \mathrm{~nm}$ (b)

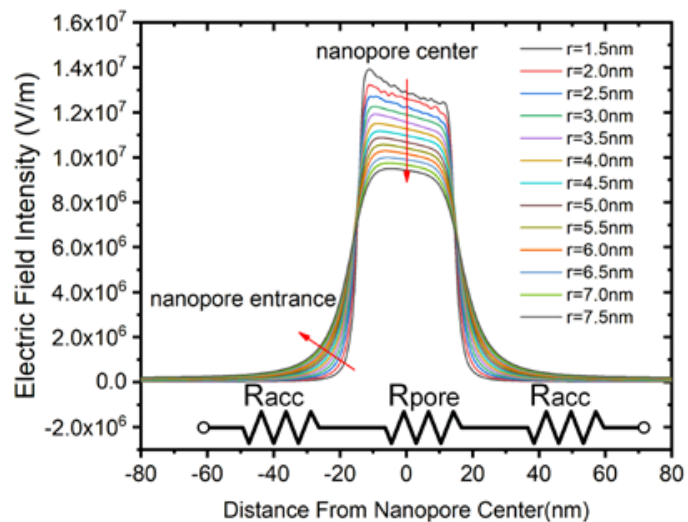

(d)

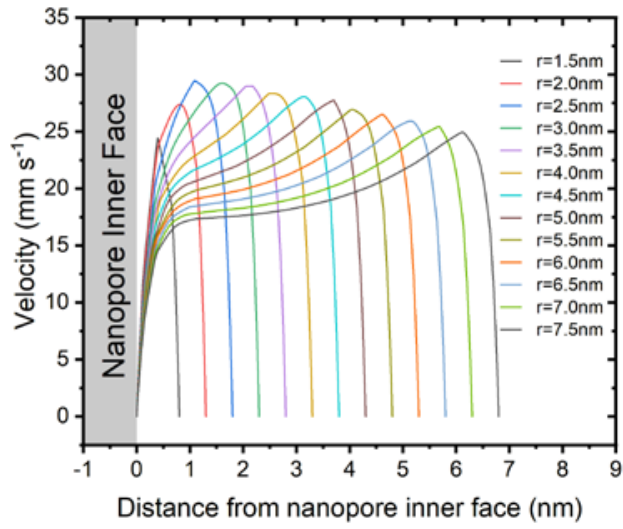

Figure S5. Electric field intensity and EOF velocity profiles inside nanopore. (a) Electric

field intensity profiles in radius of $1.5 \mathrm{~nm}, 5.0 \mathrm{~nm}$ and $7.5 \mathrm{~nm}$ nanopores. (b) Variations of electric field intensity along the axis of nanopore. Racc is access resistance and Rpore is resistance inside nanopore. (c) EOF velocity profiles for nanopores with radius of $1.5 \mathrm{~nm}$, $5.0 \mathrm{~nm}$ and $7.5 \mathrm{~nm}$ nanopores when a 50nt ssDNA located inside the pore. The black solid line represents the flow streamlines. (d) EOF velocity profiles along nanopore radial direction for different nanopores. 


\section{Reference}

(1) Briggs, K.; Charron, M.; Kwok, H.; Le, T.; Chahal, S.; Bustamante, J.; Waugh, M.; Tabard-Cossa, V. Kinetics of nanopore fabrication during controlled breakdown of dielectric membranes in solution. Nanotechnology 2015, 26 (8), 084004.

(2) Wang, Y.; Ying, C.; Zhou, W.; de Vreede, L.; Liu, Z.; Tian, J. Fabrication of multiple nanopores in a SiNx membrane via controlled breakdown. Sci Rep 2018, 8 (1), 1234.

(3) Waugh, M.; Briggs, K.; Gunn, D.; Gibeault, M.; King, S.; Ingram, Q.; Jimenez, A. M.; Berryman, S.; Lomovtsev, D.; Andrzejewski, L.; Tabard-Cossa, V. Solid-state nanopore fabrication by automated controlled breakdown. Nat Protoc 2020, 15 (1), 122-143.

(4) Wanunu, M.; Dadosh, T.; Ray, V.; Jin, J.; McReynolds, L.; Drndic, M. Rapid electronic detection of probe-specific microRNAs using thin nanopore sensors. Nat Nanotechnol 2010, 5 (11), 807-14.

(5) Ma, J.; Li, K.; Li, Z.; Qiu, Y.; Si, W.; Ge, Y.; Sha, J.; Liu, L.; Xie, X.; Yi, H.; Ni, Z.; Li, D.; Chen, Y. Drastically Reduced Ion Mobility in a Nanopore Due to Enhanced Pairing and Collisions between Dehydrated Ions. J Am Chem Soc 2019, 141 (10), 4264-4272.

(6) Lide, D. R. CRC Handbook of Chemistry and Physics2003.

(7) Ai, Y.; Liu, J.; Zhang, B.; Qian, S. Field Effect Regulation of DNA Translocation through a Nanopore. Analytical Chemistry 2010, 82 (19), 8217-8225.

(8)He, Y.; Tsutsui, M.; Fan, C.; Taniguchi, M.; Kawai, T. Controlling DNA Translocation through Gate Modulation of Nanopore Wall Surface Charges. ACS Nano 2011, 5 (7), 5509-5518.

(9) Stein, D.; Kruithof, M.; Dekker, C. Surface-charge-governed ion transport in nanofluidic channels. Phys Rev Lett 2004, 93 (3), 035901.

(10) van der Heyden, F. H.; Stein, D.; Dekker, C. Streaming currents in a single nanofluidic channel. Phys Rev Lett 2005, 95 (11), 116104.

(11) Behrens, S. H.; Grier, D. G. The charge of glass and silica surfaces. The Journal of Chemical Physics 2001, 115 (14), 6716-6721.

(12) Iler, R. K. Isolation and characterization of particle nuclei during the polymerization of silicic acid to colloidal silica. Journal of Colloid and Interface Science 1980, 75 (1), 138-148.

(13) van Hal, R. E. G.; Eijkel, J. C. T.; Bergveld, P. A general model to describe the electrostatic potential at electrolyte oxide interfaces. Advances in Colloid and Interface Science 1996, 69 (1), 31-62.

(14) Heerema, S. J.; Dekker, C. Graphene nanodevices for DNA sequencing. Nat Nanotechnol 2016, 11 (2), 127-36. 